\title{
Risk factors for secondary transmission of Shigella infection within households: implications for current prevention policy
}

Lian Boveé ${ }^{1}$, Jane Whelan ${ }^{1 *}$, Gerard JB Sonder ${ }^{1,2}$, Alje P van Dam ${ }^{3,4}$ and Anneke van den Hoek ${ }^{1,2}$

\begin{abstract}
Background: Internationally, guidelines to prevent secondary transmission of Shigella infection vary widely. Cases, their contacts with diarrhoea, and those in certain occupational groups are frequently excluded from work, school, or daycare. In the Netherlands, all contacts attending pre-school (age 0-3) and junior classes in primary school (age 4-5), irrespective of symptoms, are also excluded pending microbiological clearance. We identified risk factors for secondary Shigella infection (SSI) within households and evaluated infection control policy in this regard.

Methods: This retrospective cohort study of households where a laboratory confirmed Shigella case was reported in Amsterdam (2002-2009) included all households at high risk for SSI (i.e. any household member under 16 years). Cases were classified as primary, co-primary or SSIs. Using univariable and multivariable binomial regression with clustered robust standard errors to account for household clustering, we examined case and contact factors (Shigella serotype, ethnicity, age, sex, household size, symptoms) associated with SSI in contacts within households.

Results: SSI occurred in 25/337 contacts (7.4\%): 20\% were asymptomatic, 68\% were female, and median age was 14 years (IQR: 4-38). In a multivariable model adjusted for case and household factors, only diarrhoea in contacts was associated with SSI (IRR 8.0, 95\% Cl:2.7-23.8). In a second model, factors predictive of SSI in contacts were the age of case ( $0-3$ years (IRR case $\geq 6$ years:2.5, 95\% Cl:1.1-5.5) and $4-5$ years (IRR case $\geq 6$ years:2.2, 95\% Cl:1.1-4.3)) and household size (>6 persons ( $\left(\mathrm{RR}_{2-4}\right.$ persons $3.4,95 \%$ Cl:1.2-9.5)).

Conclusions: To identify symptomatic and asymptomatic SSI, faecal screening should be targeted at all household contacts of preschool cases ( $0-3$ years) and cases attending junior class in primary school (4-5 years) and any household contact with diarrhoea. If screening was limited to these groups, only one asymptomatic adult carrier would have been missed, and potential exclusion of 70 asymptomatic contacts $<6$ years old from school or daycare, who were contacts of cases of all ages, could have been avoided.
\end{abstract}

Keywords: Shigella, Infection control, Community-acquired infections/epidemiology, Disease transmission, Infectious, Child, Preschool

\footnotetext{
* Correspondence: jwhelan@ggd.amsterdam.nl

'Department of Infectious Diseases, Public Health Service (GGD) Amsterdam,

Nieuwe Achtergracht 100, PO Box 2200, Amsterdam 1000 CE, the

Netherlands

Full list of author information is available at the end of the article
} 


\section{Background}

Shigellosis (bacillary dysentery) is an acute intestinal infection caused by the toxin-producing gram-negative bacterium Shigella. The route of infection is faecal-oral, via the hands or through ingestion of contaminated food or water. The incubation period is typically 1-3 days. Clinical symptoms include fever, watery diarrhoea, abdominal cramps and bloody, slimy stools [1]. Disease is most severe and the case-fatality rate highest in children, the elderly and those who are immunocompromised. Case-fatality rate depends on the serotype, and is up to $20 \%$ of patients hospitalised with S. dysenteriae which occurs predominantly in less industrialised countries. In industrialised countries, S. sonnei and S. flexneri account for the majority of cases, and in the Netherlands about $75 \%$ of infections are imported, most frequently in the summer months [2]. Nationally, 300-600 cases of bacillary dysentery are reported each year, yielding an approximate annual incidence of $3.2 / 100,000$ population [2]. Secondary attack rates in households can be high [3] and infections are associated with significant morbidity and socioeconomic cost as infected individuals may be excluded from school or work pending microbiological clearance.

Guidelines for contact tracing and the control of shigellosis differ across jurisdictions. In Australia, contacts are screened routinely only in outbreak situations [4]. They are excluded from attending work or childcare, whether symptomatic or not, if they are in risk groups such as food handlers, carers or children attending childcare, until 2 successive stool samples collected a minimum of 24 hours apart are negative. In the USA, it is recommended that only symptomatic attendees and staff members in childcare centres where Shigella infection has been identified should have a faecal specimen cultured [5]. Children and staff can generally return to the child care facility $\geq 24$ hours after they are symptom free. In some US states, exclusion is continued until results of 2 stool cultures are negative for Shigella species. In the UK, contacts in risk groups are screened routinely, but microbiological clearance (two negative faecal specimens taken at intervals $\geq 48$ hours) is required for cases of S. dysenteriae, S. flexneri or S. boydii, but not for cases of S. sonnei [6].

In the Netherlands, shigellosis is notifiable by law [7]. When a case is identified, it is the responsibility of the Public Health Service (PHS) to trace contacts in order to prevent secondary infection. Until 2001, it was national policy to screen all household contacts of a Shigella case. This policy was amended based on the results of a retrospective study of shigellosis cases and their contacts reported from 1991-1998 in Amsterdam, which concluded that the highest risk of secondary transmission and hospitalisation was among children under 16 years [8]. It was recommended that contact tracing should specifically be targeted at households where children reside (hereafter, "high risk households"). In 2001, national guidelines were adjusted accordingly [9]. Since then, contact tracing is limited to faecal sampling of all household contacts if the primary case is younger than 16 years or one or more contacts in the family are younger than 16 years. If the primary case is older than 16 years and there are no younger contacts in the family, selective faecal sampling of family contacts that are careworkers or food-handlers, and those that have symptoms consistent with a Shigella infection is conducted. Under current guidelines, cases who attend childcare centres (0-3 years), or those in junior classes in primary school (4-6 years) are excluded until two consecutive faecal samples, taken at least 3 days apart and 48 hours after completion of antibiotic therapy, are confirmed negative. Furthermore, it is recommended that children of the same age who are contacts of a shigesllosis case of any age, should also be excluded from school (irrespective of symptoms) until one faecal sample is confirmed negative for Shigella [9]. The aim of this study was to determine the proportion of secondary transmissions in "high risk" households and the characteristics of primary cases and their contacts that are associated with secondary transmission, thereby to evaluate the appropriateness of current exclusion policies in relation to young children.

\section{Methods}

\section{Routine surveillance data}

A confirmed case of Shigella infection was defined as any person from whom a Shigella species was isolated from a faecal sample, reported to the PHS of Amsterdam from 2002 to 2009. In addition, case data routinely collected included age, gender, occupation, country of birth, dates of departure and return on any recent foreign trips, date of onset of illness and information about hospitalisation.

\section{Household contact study}

This was a retrospective cohort study including all occupants of "high risk" households in which a primary case of Shigella infection was reported to the PHS of Amsterdam from 2002 to 2009. A primary case was the first person in a high risk household to present with laboratory confirmed Shigella infection in a faecal sample. A high risk household was any household with more than one inhabitant including at least one child $<16$ years, where a primary case (of any age) stayed for at least one overnight, using shared toilet facilities, from the onset of symptoms to the date of notification to the PHS. Outcomes of interest were (a) any laboratory confirmed secondary infection and (b) asymptomatic laboratory confirmed secondary infection. Contacts were asked to report any symptoms experienced (diarrhoea, fever), and on what day they began 
relative to the primary case. For comparative purposes and to be consistent with previous research [8], secondary infection was defined as laboratory confirmed Shigella infection in a household contact developed $>1$ day after the primary case. If a symptomatic contact's first day of illness was $\leq 1$ day after the primary case then this contact was considered a co-primary infection and was excluded from the study. Primary cases and their contacts were also excluded if their most likely source was "Men who have sex with men" (MSM) or if the source was a common exposure to the same suspected food-source. In accordance with current guidelines, contacts were also asked to provide a faecal sample for culture by the PHS Regional Laboratory of Amsterdam, in addition to provision of general demographic information. As this research was conducted in the context of routine surveillance, no ethical approval was required.

\section{Laboratory methods}

For diagnosis of shigellosis, faecal specimens were suspended in saline and plated onto Hecto-en enteric agar. Green colonies, suspected for Shigella, were tested for fermentation of glucose and lactose using a TSI-slant and tested for urease production. Urease-negative, glucose-fermenting, lactose-nonfermenting strains were subsequently determined to the species level using API$20 \mathrm{E}$ tests (Biomerieux, Craponne, France) and agglutinated with polyvalent antisera against S.sonnei, S.flexneri, S.boydii and S.dysenteriae.

\section{Statistical analysis}

Data were analysed using Stata 11 (StataCorp LP, College Station, TX, USA). The proportion of secondary infections was the number of laboratory confirmed infected contacts divided by the total number of household contacts tested. We hypothesized that both individual characteristics of the contact (age, sex, whether symptomatic or not, hospitalized or not) as well as contextual factors in the household (household size, characteristics of the case in the household - age and sex, Shigella serotype, ethnicity, whether hospitalized or not) could be associated with secondary transmission. Age was classified in three age-groups based on school attendance: those aged $0-3$ years attending pre-school, those in junior classes in primary school aged 4-5 years, and those aged $\geq 6$ years attending senior classes in primary school. Duration from date of onset of illness to date of notification was used as a proxy measure of duration of transmission risk (i.e. prior to receipt of hygiene advice from a health professional). Univariable associations between the outcome, and individual and contextual characteristics within the household, were first tested using the Chisquared test or Fischer's exact test. As contacts and cases were clustered within households and there were a large number of clusters relative to the total sample of contacts, we used ordinary univariable and multivariable binomial regression models to obtain risk ratios with 95\% confidence intervals. These were corrected for correlation between individuals within households using clustered robust standard errors based on the HuberWhite sandwich estimator [10,11]. If the univariable association was significant at $\mathrm{p}<0.1$, variables were included in the multivariable analysis. Missing values were excluded. Finally, to compare with previous research, the annual incidence of shigellosis was estimated as the number of positive shigellosis cases per year per 100,000 residents of Amsterdam.

\section{Results}

\section{Routine surveillance data}

From 2002 to 2009, 420 primary cases of shigellosis were reported through routine surveillance. The median age was 33 years (interquartile range: $26-42)$ and $57 \%(n=241)$ were male. Eighteen percent of cases $(n=76)$ were MSM and 5 cases acquired their infection from a common suspected food-source. Of non-MSM cases born in the Netherlands, $82 \%(n=155 / 329)$ acquired their infection abroad. Of nonDutch cases who had recently travelled abroad $(n=90), 50 \%$ $(n=45)$ contracted the infection while visiting their country of origin. The most common countries where infection was acquired were Morocco $(n=52)$, Egypt $(n=38)$, India $(n=31)$, Ghana $(n=14)$, Indonesia $(n=10)$. Overall, 31\% $(n=130)$ of infections were reported in August and September. All primary cases had diarrhoea and $15 \%(n=63)$ were admitted to hospital. There were no deaths among cases.

\section{Household study: characteristics of cases, contacts and households}

MSM and those who were exposed to a common food source were excluded from the household study. Of the remaining 339 primary cases, 213 resided in non highrisk households and 24 had contacts that were selectively screened because they were symptomatic or were working as food-handlers or care-givers (Figure 1). Notably, no secondary transmissions occurred in these households. Ultimately 368 contacts related to 102 primary cases in 102 high risk households were identified. All were offered screening and 23 did not participate (Figure 1) and 8 were considered co-primary infections and were also excluded from the household study. The median household size was 4 persons (Interquartile Range,IQR: 3-5): 61 households contained 2-4 people, 24 households 5-6 people, and 17 households, $>6$ people. A greater proportion of cases (35\%) compared to contacts $(23 \%)$ were under 6 years old cases (Pearson chi-squared, $\mathrm{p}=0.03)$. The gender distribution was 


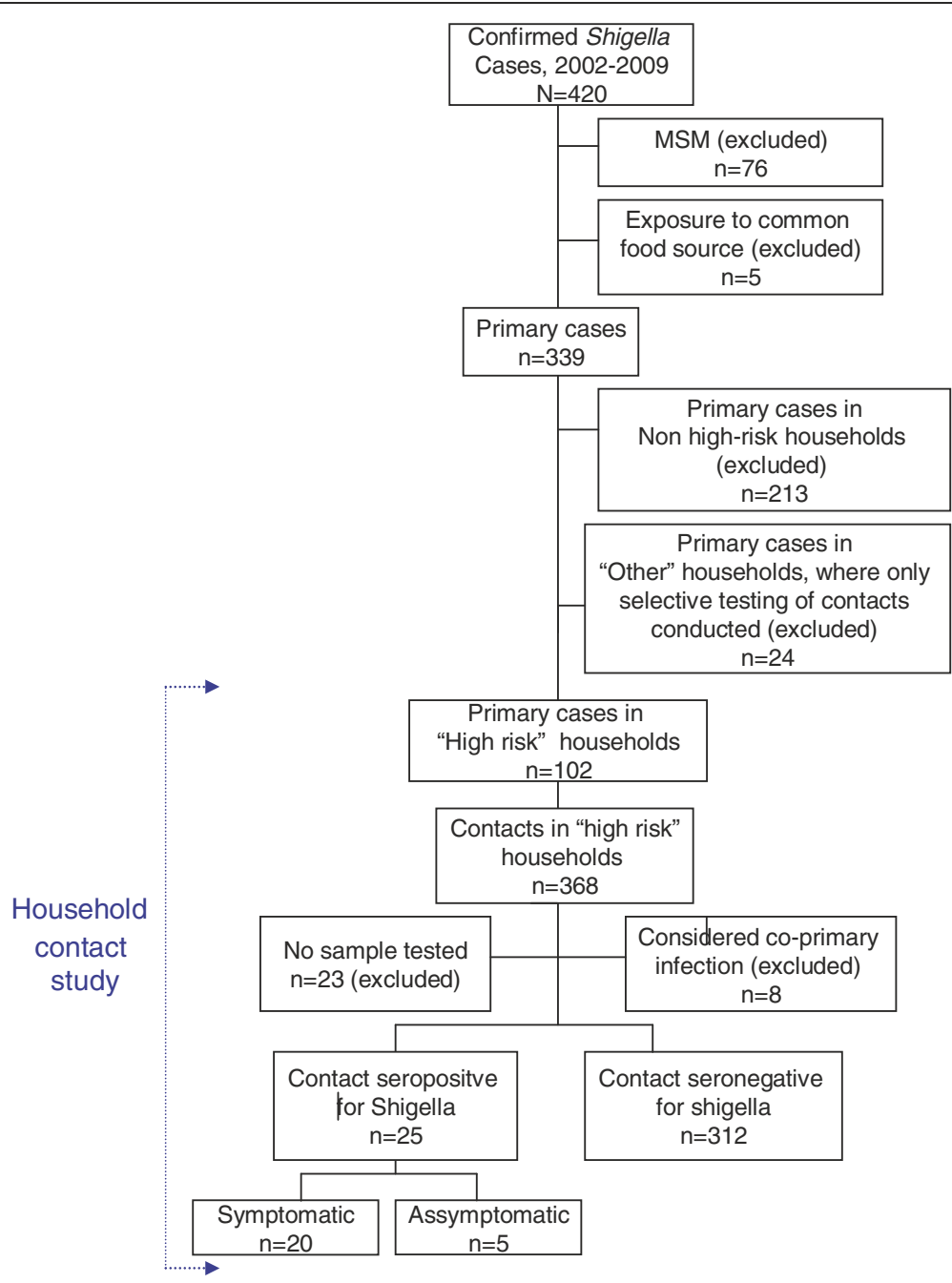

Figure 1 Flow chart of study population.

similar. Baseline characteristics of cases and household contacts are presented in Table 1.

\section{Household study: risk factors for secondary transmission}

Shigella was isolated from 25 of the remaining 337 contacts $(7.4 \%)$ of whom $68 \%(n=17)$ were female, and the median age was 14 years (IQR: $4-38$ ). The attack rate ranged from $6 \%$ in contacts aged $0-3$ years, to $17 \%$ in 4-5 year old contacts (Table 2). Ethnic backgrounds of those who acquired secondary infection were Moroccan $(n=12,48 \%)$, Dutch $(n=5,20 \%)$, Surinamese $(n=2,8 \%)$ Turkish $(n=2,8 \%)$ Croatian $(n=2,8 \%)$ or "other" $(n=2$, $8 \%)$. One 8 -month old contact was admitted to hospital and there were no deaths. Over half of secondary infections $(56 \%, n=14)$ were associated with primary cases who were under 6 years old. The nature of the positive contact's relationship to the primary case was as follows: sibling $(n=7$, median age 4 , IQR: $2-12)$, mother $(n=7)$, father $(n=3)$, offspring $(n=2)$, or other family contact
( $n=6$, median age 5.5, IQR: 4-14). Statistically there was no association between secondary infection and the nature of the contact's relationship to the primary case, and we did not find any difference in age between siblings who were secondary versus non-secondary cases (mean age 8.1 \& 10.6 respectively, $\mathrm{p}=0.502$ ). Thirteen primary cases were associated with one positive household contact and 6 primary cases were associated with 2 positive household contacts. No factor was identified that was associated with $>1$ secondary transmission. Overall, the mean annual incidence of Shigella infection in Amsterdam from 2002-2009 was 7.7/100,000 persons.

At the univariable level (Table 2), secondary transmission was more likely to occur if the household contact was aged 4-5 years or if they had diarrhoea, or in turn if the primary case was aged 4-5 years old, or in large households ( $>6$ persons). In the multivariable model, the only significant correlation observed, was between secondary infection and the presence of diarrhoea in 
Table 1 Baseline characteristics of primary Shigella cases $(n=102)$ and related contacts $(n=337)$ in high risk households, Amsterdam, The Netherlands 2002-2009

\begin{tabular}{|c|c|c|c|c|c|c|}
\hline \multirow{2}{*}{\multicolumn{2}{|c|}{ Characteristics }} & \multicolumn{2}{|c|}{$\begin{array}{l}\text { Primary cases } \\
(n=102)\end{array}$} & \multicolumn{2}{|c|}{$\begin{array}{l}\text { Household contacts } \\
\qquad(\mathrm{n}=337)\end{array}$} & \multirow[t]{2}{*}{$\mathrm{p}$ value } \\
\hline & & $\mathrm{n}$ & $\%$ & $\mathrm{n}$ & $\%$ & \\
\hline \multicolumn{7}{|l|}{ Age group } \\
\hline & $0-3$ & 20 & 20 & 48 & 14 & \\
\hline & $4-5$ & 15 & 15 & 19 & 9 & \\
\hline & $>6$ & 67 & 66 & 260 & 77 & 0.030 \\
\hline \multicolumn{7}{|l|}{ Gender } \\
\hline & female & 53 & 52 & 171 & 51 & \\
\hline & Male & 49 & 18 & 166 & 49 & 0.802 \\
\hline \multicolumn{7}{|l|}{ Country of birth } \\
\hline & Netherlands & 30 & 29 & 91 & 27 & \\
\hline & Western, other & 3 & 3 & 6 & 2 & \\
\hline & Non-western & 70 & 68 & 236 & 71 & \\
\hline \multicolumn{7}{|l|}{ Diarrhoea } \\
\hline & Yes & 102 & 100 & 87 & 26 & \\
\hline & No & 0 & 0 & 228 & 68 & \\
\hline & Unknown & 0 & 0 & 22 & 7 & \\
\hline \multicolumn{7}{|l|}{ Shigella isolated } \\
\hline & Yes & 102 & 100 & 25 & 7 & \\
\hline & No & 0 & 0 & 312 & 86 & \\
\hline \multicolumn{7}{|l|}{ Shigella serotype } \\
\hline & S. sonnei & 57 & 56 & 15 & 4 & \\
\hline & S. flexneri & 36 & 35 & 8 & 2 & \\
\hline & S. boydii & 6 & 6 & 2 & 1 & \\
\hline & S. dysenteriae & 3 & 3 & 0 & 0 & \\
\hline & Negative & 0 & 0 & 312 & 93 & \\
\hline \multicolumn{7}{|l|}{ Months reported } \\
\hline & Dec-Jan & 9 & 9 & 46 & 14 & \\
\hline & Feb-Mar & 6 & 6 & 15 & 4 & \\
\hline & Apr-May & 9 & 9 & 25 & 7 & \\
\hline & Jun-Jul & 8 & 8 & 20 & 6 & \\
\hline & Aug-Sep & 51 & 50 & 168 & 50 & \\
\hline & Oct-Nov & 19 & 19 & 63 & 19 & \\
\hline \multicolumn{7}{|l|}{ Hospitalised } \\
\hline & No & 22 & 22 & 1 & 0 & \\
\hline & Yes & 80 & 78 & 336 & 100 & \\
\hline
\end{tabular}

the household contact. We re-ran the multivariable model without diarrhoea as a variable (data not shown in Table 2). Independent predictors of secondary infection in contacts were then if the case was aged 0-3 years (Incidence Rate Ratio, $\mathrm{IRR}_{\text {case } \geq 6 \text { years }}: 2.5$, 95\% CI:1.1$5.5)$ or 4-5 years (IRR case $\geq 6$ years: $2.2,95 \% \mathrm{CI}: 1.1-4.3)$ and households with more than 6 persons (IRR2-4 persons 3.4, 95\% CI:1.2-9.5). Contact characteristics were not significant in this model. Overall, 5/25 secondary infections were asymptomatic (20\%). These were persons aged 5 and 8 years old, and three were aged $>30$ years. When contacts who were symptomatic and positive for Shigella were excluded $(n=20)$, we did not find any association between the age of the contact ( $\geq 6$ years old (ref.) versus $<6$ years) and the risk of asymptomatic infection (IRR:0.9, 95\% CI:0.2-5.0). 
Table 2 Univariable and multivariable risk factors for secondary Shigella transmission to 337 contacts within 102 households*, Amsterdam, The Netherlands 2002-2009

\begin{tabular}{|c|c|c|c|c|c|c|c|c|c|c|c|}
\hline \multirow[t]{3}{*}{ Exposure } & \multirow{3}{*}{$\begin{array}{c}\begin{array}{c}\text { Total no. of household } \\
\text { contacts }\end{array} \\
\mathrm{N}\end{array}$} & \multicolumn{2}{|c|}{$\begin{array}{c}\text { Any secondary } \\
\text { transmission }\end{array}$} & \multicolumn{4}{|c|}{ Univariable } & \multicolumn{4}{|c|}{ Multivariable } \\
\hline & & \multirow{2}{*}{$\mathbf{n}$} & \multirow{2}{*}{$\%$} & \multirow{2}{*}{ RR } & \multicolumn{2}{|c|}{$95 \% \mathrm{Cl}$} & \multirow{2}{*}{$p$ value } & \multirow{2}{*}{ RR } & \multicolumn{2}{|c|}{$95 \% \mathrm{Cl}$} & \multirow{2}{*}{$\mathrm{p}$ value } \\
\hline & & & & & Lower & Upper & & & Lower & Upper & \\
\hline \multicolumn{12}{|c|}{ Contact characteristics ( $n=337$ contacts) } \\
\hline Total & 337 & 25 & 7.4 & n/a & $\mathrm{n} / \mathrm{a}$ & $\mathrm{n} / \mathrm{a}$ & $\mathrm{n} / \mathrm{a}$ & n/a & n/a & $\mathrm{n} / \mathrm{a}$ & n/a \\
\hline \multicolumn{12}{|l|}{ Age-group } \\
\hline$\geq 6$ years & 260 & 17 & 6.5 & ref. & ref. & ref. & ref. & ref. & ref. & ref. & ref. \\
\hline $0-3$ & 48 & 3 & 6.3 & 1.0 & 0.3 & 3.3 & 0.943 & 1.0 & 0.3 & 3.2 & 0.979 \\
\hline $4-5$ & 29 & 5 & 17.2 & 2.6 & 1.0 & 6.8 & 0.046 & 1.3 & 0.6 & 3.0 & 0.527 \\
\hline \multicolumn{12}{|l|}{ Gender } \\
\hline Female & 171 & 17 & 9.9 & ref. & ref. & ref. & ref. & & & & \\
\hline Male & 166 & 8 & 4.8 & 0.5 & 0.2 & 1.2 & 0.109 & & & & \\
\hline \multicolumn{12}{|l|}{ Country of birth } \\
\hline Netherlands / Other Western & 97 & 7 & 7.2 & ref. & ref. & ref. & ref. & & & & \\
\hline Other & 236 & 18 & 7.6 & 1.1 & 0.5 & 2.3 & 0.890 & & & & \\
\hline \multicolumn{12}{|l|}{ Diarrhoea } \\
\hline No & 228 & 5 & 2.2 & ref. & ref. & ref. & ref. & ref. & ref. & ref. & ref. \\
\hline Yes & 87 & 20 & 23.0 & 10.5 & 3.7 & 29.9 & $<0.001$ & 8.0 & 2.7 & 23.8 & $<0.001$ \\
\hline \multicolumn{12}{|l|}{ Hospitalised } \\
\hline No & 336 & 24 & 7.1 & ref. & ref. & ref. & ref. & & & & \\
\hline Yes & 1 & 1 & 100.0 & - & - & - & - & & & & \\
\hline
\end{tabular}

\section{Household factors ( $n=102$ households)}

Household size

\begin{tabular}{lccccccccccc} 
2-4 persons (in 61 households) & 128 & 5 & 3.9 & ref. & ref. & ref. & ref. & ref. & ref. & ref. & ref. \\
5-6 persons (in 24 households) & 96 & 5 & 5.2 & 1.3 & 0.4 & 4.8 & 0.658 & 1.4 & 0.4 & 4.6 & 0.570 \\
>6 persons (in 17 households) & 113 & 15 & 13.3 & 3.4 & 1.3 & 8.7 & 0.011 & 3.1 & 0.9 & 10.1 & 0.064 \\
\hline
\end{tabular}

Age of primary case in household

\begin{tabular}{lccccccccccc}
$\geq 6$ years & 218 & 11 & 5.1 & ref. & ref. & ref. & ref. & ref. & ref. & ref. & ref. \\
$0-3$ & 58 & 6 & 10.3 & 2.1 & 0.8 & 5.5 & 0.152 & 2.6 & 1.0 & 7.1 & 0.061 \\
$4-5$ & 61 & 8 & 13.1 & 2.6 & 1.2 & 5.7 & 0.018 & 1.9 & 0.8 & 4.5 & 0.163 \\
\hline
\end{tabular}

Gender of primary case in household

\begin{tabular}{lccccccc} 
Female & 174 & 9 & 5.2 & ref. & ref. & ref. & ref. \\
$\quad$ Male & 163 & 16 & 9.8 & 1.9 & 0.9 & 4.1 & 0.103 \\
\hline $\begin{array}{l}\text { Primary case in household hospitalised } \\
\text { No }\end{array}$ & 264 & 21 & 8.0 & ref. & ref. & ref. & ref. \\
$\quad$ Yes & 73 & 4 & 5.5 & 1.0 & 0.9 & 1.2 & 0.405 \\
\hline
\end{tabular}

Time from date of onset to notification of primary case

$\begin{array}{lccccccc}\leq 1 \text { week } & 95 & 9 & 9.5 & \text { ref. } & \text { ref. } & \text { ref. } & \text { ref. } \\ 1-3 \text { weeks } & 146 & 8 & 5.5 & 0.6 & 0.2 & 1.5 & 0.262 \\ >3 \text { weeks } & 96 & 8 & 8.3 & 0.9 & 0.4 & 2.0 & 0.761\end{array}$

*The unit of analysis is the individual contact $(n=337)$ and cluster characteristics are at the household level: household size, age and gender of the primary case in the household, whether they were hospitalised and time from date of onset to notification of primary case, as above.

\section{Discussion}

The proportion of secondary transmissions of laboratory confirmed Shigella infection in high risk household contacts of primary cases reported in Amsterdam from 2002 to 2009 was $7.4 \%$. Though not directly comparable to our study, similar intra-familial or household secondary transmission rates of Shigella have been reported in studies conducted in outbreak settings internationally $[12,13]$. This rate is also similar to that of $8 \%$ reported by Vermaak et al. [8] in Amsterdam from 1992-1998. In 
our study, only households with contacts considered to be at high risk of secondary infection were included. As this represented a smaller denominator population than in Vermaak et al. [8], we had expected to find a relative increase in the rate of secondary infection. One explanation is that we underestimated the secondary attack rate and that (unscreened) positive asymptomatic cases in non-high risk households were missed. We consider this unlikely as in Vermaak et al. [8] this accounted for only 3 extra cases over 8 years. An alternative explanation is that hygiene standards in households in the Netherlands and abroad have improved over time, reducing the potential for secondary spread. The majority of Shigella infections are imported, but recent national research has shown that between 1995 and 2006 there was a significant reduction in the incidence of Shigella infection among travelers from the Netherlands which was related to improved hygiene standards in the countries visited [14]. Despite a doubling in the annual number of travelers to (sub)tropical countries from about 1 million in 1999 to 2 million in 2007 [15], the incidence of shigellosis in Amsterdam has remained relatively static at 8/ 100,000 in 1998 [8], and 7.7/100,000 annually from 2002-2009.

Where outbreaks of shigellosis have occurred in nurseries and schools, they have generally been attributable to children with diarrhoea who visited the institution or those who returned to school before being cultureconfirmed negative $[12,16]$. Outbreaks have been brought under control by excluding young shigellosis cases from school or daycare where supervision of a child's hygiene may be inconsistent, pending microbiological clearance $[17,18]$. In our first multivariable model, the only factor independently associated with Shigella positivity in a household contact was diarrhoea, irrespective of the age of the case or of the contact. The current policy, that all contacts with diarrhoea should be investigated for the presence of the bacterium Shigella, is therefore supported.

In the second multivariable model which examined predictors of secondary infection in contacts, preschool cases aged $0-3$, those in junior classes in primary school aged 4-5 years, and those in large households were more likely to transmit (both symptomatic and asymptomatic) infection. Typically, young children who use the toilet independently but have limited understanding of good hand- and toilet-hygiene may be particularly susceptible to transmitting secondary infection. We did not find any increased risk among siblings of cases, or their mothers compared to other household contacts however, unlike similar research examining household transmission of E.Coli 0157 [19]. Based on our findings, screening of all contacts of cases who are under 6 years is also recommended. In fact, had faecal screening been limited to household contacts of cases who were under 6 years old and contacts with diarrhoea as we suggest, $96 \%$ of secondary cases would have been detected and only one asymptomatic adult carrier would have been missed. Additional faecal sampling of 164 contacts would not have been required.

The policy in the Netherlands of excluding all contacts under 6 years old pending a single negative faecal culture sample is generally not supported by our findings. In the multivariable models, the age of the contact was not independently associated with secondary Shigella infection and we found no association between young age of contact ( $<6$ years old) and a risk of asymptomatic infection. In our study over the 8 year period, 70 asymptomatic children under 6 years old were potentially excluded from school or daycare pending microbiological clearance. This yielded only one asymptomatic infection. Although a formal cost-benefit analysis would be necessary to systematically compare costs, given considerable practical difficulties and low added value, the policy of excluding young children who are asymptomatic contacts of a case with shigellosis should be revisited.

There were a number of study limitations: firstly, we were unable to examine the risk of asymptomatic secondary transmission in low risk households, however among those at highest risk in these households who were screened (i.e. those who were symptomatic, or were careworkers or food-handlers) no secondary transmissions occurred. Secondly, we defined a secondary infection in a household contact as one that developed $>1$ day after the primary case. Had we used a more conservative definition (e.g. $\geq 3$ days, based on the median incubation period), one additional case would have been reclassified, representing a secondary attack proportion of $7.2 \%$. The associations at both univariable and multivariable level would not change however. Thirdly, there was a delay between date of onset of illness and date of notification of $>3$ weeks in $28 \%$ of cases. Recall bias is therefore likely, and cases and contacts may have reported estimated rather than precise dates of onset of illness. Ultimately, delay in reporting was not associated with secondary transmission of infection. Fourthly, culture was used for diagnosis of shigellosis, though it is recommended that the sample is submitted within 24 hours, false negatives may have occurred. The use of more sensitive molecular methods [20] might have revealed more cases of secondary transmission. Finally, given the low proportion of secondary transmissions, it is possible some differences may have been undetected due to insufficient power.

\section{Conclusions}

In conclusion, guidelines relating to screening and exclusion of contacts in order to prevent secondary transmission of Shigella infection vary widely internationally. Prevention of secondary transmission through education 
and promotion of hand washing and strict hygiene practices in affected households remain the mainstay of Shigella control $[5,6,21]$. To identify symptomatic and asymptomatic SSI, all household contacts of young Shigella cases ( $<6$ years old) and contacts with diarrhoea should be screened. Exclusion of cases and contacts with diarrhoea from work, school and daycare remains important to prevent spread, but our findings do not support exclusion of asymptomatic child contacts under 6 years.

\section{Competing interests}

No competing interests are declared by any of the authors.

\section{Authors' contributions}

LB and JW contributed equally as first authors of the paper including data cleaning, analysis, interpretation and writing of the manuscript. A.P. van Dam conducted the laboratory testing and contributed to the methodology and discussion sections and provided critical review of the paper. GS and A. van den Hoek conceived the research question, provided critical review and guidance throughout and are ultimately responsible for the research. All authors read and approved the final manuscript.

\section{Acknowledgements}

This research project was supported by a grant provided by the regional programme budget of the National Centre for Infectious Disease control in the Netherlands. We would also like to thank the nurses of the department of infectious diseases in Amsterdam who collected and recorded the data of cases and their contacts, and M. Siebbeles (MD) who reviewed the case charts.

\section{Author details}

'Department of Infectious Diseases, Public Health Service (GGD) Amsterdam, Nieuwe Achtergracht 100, PO Box 2200, Amsterdam 1000 CE, the Netherlands. ${ }^{2}$ Department of Internal Medicine, Academic Medical Center, Division of Infectious Diseases, Tropical Medicine and AIDS, Meibergdreef 9 , Amsterdam 1105 AZ, The Netherlands. ${ }^{3}$ Public Health Laboratory, Public Health Service (GGD) Amsterdam, Nieuwe Achtergracht 100, PO Box 2200, Amsterdam $1000 \mathrm{CE}$, the Netherlands. ${ }^{4}$ Department of Medical Microbiology, Onze Lieve Vrouwe Gasthuis (OLVG Hospital), Postbus 95500, Amsterdam 1090 HM, the Netherlands.

Received: 8 June 2012 Accepted: 5 December 2012

Published: 12 December 2012

\section{References}

1. American Public Health Association: Control of Communicable Diseases Manual. 19th edition. Washington, DC: American Public Health Association; 2008.

2. van Pelt W, de Wit MA, Wannet WJ, Ligtvoet EJ, Widdowson MA, et al: Laboratory surveillance of bacterial gastroenteric pathogens in The Netherlands, 1991-2001. Epidemiol Infect 2003, 130:431-441.

3. Dupont H: Shigella species. In Mandell, Douglas and Benett's Principles and Practice of Infectious D/seases. 6th edition. Edited by Mandell G, Benett J, Dolin R. Philadelphia: Churchill Livingstone; 2005:2655-2661.

4. Queensland Government: Shigella infection (Shigellosis): Queensland Health Guidelines for Public Health Units. Brisbane, Queensland, Australia: Queensland Government; 2010.

5. American Academy of Pediatrics: In Red Book: 2009 report of the Committee on Infectious Diseases. 28th edition. Edited by Pickering L, Baker C, Long S, McMillan J. Elk Grove Village, IL: American Academy of Pediatrics; 2009:593-596.

6. PHLS Advisory Committee on Gastrointestinal Infections: Preventing person-to-person spread following gastrointestinal infections: guidelines for public health physicians and environmental health officers. Commun Dis Public Health 2004, 7(4):362-384.

7. National Institute of Public Health and the Environment (RIVM): Reporting of infectious diseases in accordance with the Public Health Act. Bilthoven:
National Institute of Public Health and the Environment (RIVM) [In Dutch]; 2008.

8. Vermaak M, Langendam M, van den Hoek J, Peerbooms PG, Coutinho R: Shigellosis in Amsterdam, 1991-1998: prevention and results of contact tracing [in Dutch]. Ned Tijdschr Geneeskd 2000, 144:1688-1690.

9. National Center for the coordination of Infectious Disease Control (LCI): $L C$ Directive: Shigellosis. Bilthoven: National Institute of Public Health and the Environment (RIVM); 2011.

10. Rogers WH: Regression standard errors in clustered samples. Stata Technical Bulletin 13: 19-23. Reprinted in Stata Technical Bulletin Reprints 1993, 3:88-94.

11. Williams RL: A note on robust variance estimation for cluster-correlated data. Biometrics 2000, 56:645-646.

12. De Schrijver K, Bertrand S, Gutierrez Garitano I, Van den Branden D, Van Schaeren J: Outbreak of Shigella sonnei infections in the Orthodox Jewish community of Antwerp, Belgium, April to August 2008. Euro Surveill 2011, 16(14):pii=19838.

13. Khan Al, Talukder KA, Huq S, Mondal D, Malek MA, Dutta DK, et al: Detection of intra-familial transmission of shigella infection using conventional serotyping and pulsed-field gel electrophoresis. Epidemiol Infect 2006, 134(3):605-611.

14. Baaten GG, Sonder GJ, Van Der Loeff MF, Coutinho RA, Van Den Hoek A: Faecal-orally transmitted diseases among travelers are decreasing due to better hygienic standards at travel destination. J Travel Med 2010, 17(5):322-328

15. Dutch Tourist Board and NIPO Research: Continuous Holiday Survey [in Dutch]. Leidschendam: Dutch Tourist Board and NIPO research; 1999-2007.

16. Jonsson J, Alvarez-Castillo Mdel C, Sanz JC, Ramiro R, Ballester E, Fernanez $M$, et al: Late detection of a shigellosis outbreak in a school in Madrid. Euro Surveill 2005, 10(10):268-270.

17. Mohle-Boetani JC, Stapleton M, Finger R, Bean NH, Poundstone J, Blake PA, et al: Communitywide shigellosis: control of an outbreak and risk factors in child day-care centers. Am J Public Health 1995, 85(6):812-816.

18. Arvelo W, Hinkle C, Nguyen T, et al: Transmission risk factors and treatment of pediatric shigellosis during a large daycare centerassociated outbreak of multidrug resistant Shigella sonnei. Implications for the management of shigellosis outbreaks among children. Pediatr Infect Dis J 2009, 28:976-980

19. Werber D, Mason B, Evans M, Salmon R: Preventing household transmission of Shiga Toxin-producing Escherichia coli 0157 infection: Promptly separating siblings might be the key. CID 2008, 46:1189-1196.

20. de Boer RF, Ott A, Kesztyus B, Kooistra-Smid AM: Improved detection of five major gastrointestinal pathogens by use of a molecular screening approach. J Clin Microbiol 2010, 48(11):4140-4146.

21. Centers for Disease Control (CDC): Community outbreaks of shigellosis--United States. MMWR Morb Mortal Wkly Rep 1990 39(30):509-513. 19

doi:10.1186/1471-2334-12-347

Cite this article as: Boveé et al:: Risk factors for secondary transmission of Shigella infection within households: implications for current prevention policy. BMC Infectious Diseases 2012 12:347.

\section{Submit your next manuscript to BioMed Central and take full advantage of:}

- Convenient online submission

- Thorough peer review

- No space constraints or color figure charges

- Immediate publication on acceptance

- Inclusion in PubMed, CAS, Scopus and Google Scholar

- Research which is freely available for redistribution 